\title{
Time, Culture and Identity: A Digital and Creative Professional's Perspective on Interpreting Historical Clocks in Museum Environments
}

\author{
Dominic ROBSON ${ }^{1}$
}

(Goldsmiths, University of London, London SE14 6NW, UK)

\begin{abstract}
Digital media offer unique opportunities for museums to bring to life the secrets and stories of their historical collections. To bring insight into the process of developing digital media exhibits, this paper presents the perspective of a creative practitioner in approaching technology- and media-based interpretation for collection objects. It follows the Time, Culture and Identity digital workshop held in Beijing in October 2019, which explored and shared ideas about collaborative research and interdisciplinary practice in digital interpretation between academics, institutions, creative practitioners, and developers. Following the direction of the workshop, the paper takes as its focus the clocks and automatons of the imperial collection at the Palace Museum in Beijing. Observations are based on the author's practice-led experience in running a design studio, Harmonic Kinetic, developing new media exhibits using digital technology and audiovisual media for museums, galleries, and exhibitions in the UK, including the Science Museum, V\&A, Barbican, Tate, and the Tower of London. Taking a broad interaction-design-led outlook, the paper explores a personal design perspective for developing interpretive content and considers the particular opportunities and approaches these historical devices suggest. The paper concludes with a final section that reviews the process and reflects on outcomes from the Time, Culture and Identity digital workshop. This explored possibilities for an interpretive exhibit on the Country Scene clock from the Palace Museum collection.
\end{abstract}

Keywords: object interpretation, digital media, exhibition design, mechanical clocks, automatons, Palace Museum

\section{Introduction}

$\mathrm{T}$ he history of clocks and timekeeping offers an exciting and creative space to work for developing interpretation. It brings together a huge range of themes spanning

Received: March 31, 2020. Revised: September 11, 2020.

1 Research interests: Interaction design, museum object interpretation, visitor engagement, spaces for communication. Email: d.robson@gold.ac.uk 
everything from materials science and engineering to economics and political philosophy. In human culture, the concept of time is almost universally experienced and acknowledged in some form, and yet how it is adopted, applied, or ignored remains very diverse. Our artifacts of time offer an incredible lens through which to explore human culture in all its forms. The clock as object in its own right brings its own themes and stories. The clocks, or perhaps more accurately the automatons, of the Palace Museum distinctly demonstrate this in becoming objects at the center of a unique historic cultural exchange.

This paper looks at how digital technology can be applied to object interpretation, and follows a participatory digital workshop as part of the Time, Culture and Identity research project, a collaboration between the Science Museum in London, the Palace Museum, Beijing Jiaotong University, and the Institute for the History of National Sciences in Beijing. The project seeks to build creative partnerships between China and the UK while using it to explore cultural exchange and develop cross-cultural approaches for visitor interpretation of historic objects.

\section{Digital technology within the museum environment}

As part of the Time, Culture and Identity project, the digital workshop held in Beijing in October 2019 included presentations from a range of Chinese and UK-based creative practitioners and academics, and explored a range of methodologies, techniques, and tools used in the interpretation of cultural heritage. A key aim of the project was to explore how Chinese and British museum visitors each encounter historical scientific collections.

New media offer powerful means to engage museum visitors in rich stories and experiences around collection objects. There is now an expectation for digital content in its many forms - media environments, interactive displays, physical interfaces, and so on - to be a key part of any museum visit. Developments in technology and reductions in the cost of media hardware give museums a wide range of possibilities to play with large-scale visual displays, augmented reality, serious gaming, immersive environments, data visualizations, interactive tables, animated models, and projection mapping across spaces and objects. From a visitor experience perspective, new media can create displays that inspire wonder through viewing spectacle as well as providing direct engagement with content through hands-on interaction.

For museums, creating digital interpretation is not as straightforward as it once was. In 2020, 3.5 billion people own a smartphone (Newzoo 2019), a figure approaching 50\% of the world's population. Ten years ago, the interactive digital content offered by museums was novel, but in an era in which smartphone use can average over three hours a day (Wurmser 2019), it is now firmly part of our everyday existence. These 
developments raise several issues.

With an increasing concern about the dominance of technology and its impact, particularly on young children and adolescents, how museums choose to apply digital technology to pursue their core mission is critical. How do museums maintain a balance in using digital content to work with and support collections without it merely leading to more screen time, ultimately detracting attention from the object displays? How can they develop engaging digital content without having the production budgets to compete with leading games and software producers?

From all these perspectives, the museum's role in creating engaging digital content is a big challenge. Museums need to ensure that they are not trying to compete with or duplicate material that exists elsewhere and is readily accessible; they should resist the impulse to keep up with technological developments for the sake of it. Instead they need to innovate appropriately with the modest budgets available and the unique assets they do have, such as amazing object collections; big, beautiful public spaces; and an excited, engaged audience.

\subsection{It's not magic}

From one perspective, however, there is nothing magic about digital technologies. They offer us exciting, novel means and media forms for interpreting historical objects - old stories told with new technology. But like any media, digital media come with their own sets of possibilities and constraints. Compared with older forms of media, for example, the printed object label, digital media have clear strengths and weaknesses. The object caption is not particularly dynamic, but it is simple, offers clear, unambiguous information that is readily understood (as long as you can read the language in print), it is relatively cheap and is unlikely to stop working. As such it is perfect for delivering basic information very reliably and so is still largely used for object displays around the world. However, it is not so good at delivering anything that cannot be summarized in a few sentences, so remains constrained in communicating detailed stories and ideas. Digital technologies have amazing capacity to tell these stories in an engaging way, but are very intensive to develop and feature hardware that needs turning on, maintaining, and replacing. They require a completely different level of commitment from exhibition developers. Understanding where strengths and weaknesses lie with any technology is critical, allowing us to choose the most appropriate one to use as a tool for creating interpretation.

\section{What - making the invisible visible}

So how do developers generate a particular idea for an exhibit? It is implicit to the notion of interpretation that an important aspect of the curator's and exhibit 
developer's role is to reveal those things that the object does not present in its immediate physical presence - to make the invisible visible.

This is a broad aspiration, and it can range from highlighting the historical and social background of objects, to exploring narrative details of their physical materiality and functionality. Blyth and Prugnon (2015) argue that while scientific objects do carry some intrinsic appeal with their appearance and function, there is the opportunity to reach and engage a much wider audience "if we can reveal captivating stories around them." As an idea this can generate constructive possibilities for the developer. What are the most interesting factors to uncover? What are the stories most likely to engage the public?

\subsection{Telling the story of the history around an object}

- What was the social, historic, or economic context of development? Why did this object emerge?

- How was it developed? Who made it?

- What are the different processes involved in making it?

- What was the context of consumption? Who used it, who owned it, where was it kept, and who was responsible for its maintenance?

- Does it have its own unique story beyond the grander historical themes?

The clocks of the Palace Museum carry a strong historical story, but the most interesting perspective is arguably the cultural exchange they created between Europe and the Chinese imperial court. The huge fascination of the emperors of the Ming and Qing dynasties with European clock technology and the novelty it offered them is a rich story of the connection between the very different worlds of Chinese imperial life and European engineering in the seventeenth and eighteenth centuries.

In describing the interpretation approach adopted for developing the Information Age gallery at the Science Museum, the principal curator, Blyth (2015), reflected on the decision to avoid presenting grand narratives in favor of using multiple narratives to more fully explore the social, economic, and cultural contexts that form the backdrop of historical technologies. This strategy enables a rich set of perspectives that consider not just the inventor or maker of an object at the point of invention, but the range of people involved in its existence, across a longer timescale: the craftspeople, the audiences, the operators, the courtiers, the keepers.

From audience research as part of the Time, Culture and Identity research project: "Accompanying accessible human stories help to stimulate empathy and engagement. The characters and connections may have differed between China and the UK, but the power of an accessible hero was evident throughout. Findings suggest that audiences across the UK and China all welcome human stories" (Chung 2020). 


\subsection{The collection status of the object}

- Where does the value in the object lie? Why is it in the collection?

- How does it compare with other objects in the collection?

- Who looks after the objects now?

This is a contemporary view on the object, and positions a particular group of clocks and automatons in the history of clockmaking and mechanical crafts across centuries. Clockwork has been very successful as a technology that with careful maintenance can work across centuries. The oldest working clocks are over five hundred years old. So, within the story of collections this is also the very human story of the conservators and technicians who have kept the mechanical systems in working order. In China the human story came in the form of Mr. Wang Jin, the horological conservator of the Forbidden City who became a media celebrity following a China Central Television documentary, Masters in the Forbidden City. Instead of taking a historical perspective, the documentary chose to tell the contemporary story of the clocks through the everyday life and work of Mr. Wang Jin, their restorer, successfully inspiring a renewed public interest in the collection.

\subsection{Presenting views of the object that are not immediately possible in the context of the museum}

- Showing details normally hidden from view, for example, showing the internal mechanisms of the automatons.

- Showing the object functioning in everyday operation, for example, showing a clock working.

- Visualizing important concepts around the object, schematically illustrating how things work.

These last opportunities are particularly relevant for mechanical devices in which much of the engineering wonder may be hidden from the visitors' view or exist at a scale that is hard for visitors to appreciate without zooming in to see the detail. Much of the magic and uniqueness of the Country Scene clock from the Palace Museum collection lies in the beauty and imagination of the clockwork automaton, something that is impossible to exhibit as a permanent working object. An important starting point then is to explore how the spectacle and experience of the clock's automated mechanical sequence can be revealed and presented to the visitor standing in front of the static object.

\section{How-developing ideas}

The exhibit developer's role is then to look at how this content can be taken and 
converted into a form in which it can engage a broad public in a practical form. This is an interaction design challenge. Most interpretation exhibits will start with a creative brief written by the exhibition and curatorial team which defines the scope of the project and sets its important parameters. From this, exhibit developers will generate a series of simple creative concepts exploring different possibilities for the exhibit. So, what are the assets, the ideas, the techniques that help guide the exhibit developer to move forwards?

\subsection{Thinking about the visitor experience}

One useful method is to apply an experience-led model to think about how visitors experience an overall exhibition space and the content within it. In his history of special effects, Klein (2004) explores the concept of scripted spaces-environments carefully designed around the visitor's journey in which "the audience walks into the story." The experience model considers the different stages in the journey through the museum space from the perspective of a first-time visitor, to try and see it through their eyes. It imagines how they first perceive the display, how it catches their attention within the busy and noisy exhibition environment.

Rounds (2004) takes this idea further. Adopting approaches from behavioral ecology, he proposes that the curiosity-driven visitor forages an exhibition to maximize the "Total Interest Value" of their visit. While their journey appears to be random and unfocused, the visitor is using simple heuristic systems to navigate the "Interest Landscape" of the exhibition to "satisfy and pique their curiosity."

From these kinds of perspectives we can think about how the object and its digital interpretation need to work as a display to draw attention and interest. At a distance, the priority is to initiate engagement with the object and its environment. As the visitor draws closer, the display needs to be spatially legible; the object and interpretation must be arranged in a way that enables the visitor to understand what they are looking at and what they should do if the display is in any way interactive. Once the visitor's attention is engaged, what is the mechanism for delivering the interpretation content? Is the medium or activity engaging enough to hold the visitor's attention long enough before they want to move on to something new? Museums are packed full of the curious, the weird, and the wonderful, all competing for attention. For most visitor groups, anything more than a minute spent with a single exhibit can count as a long "dwell" time.

Using an experience model also helps us think about different visitor groups and consider what will work for each of them. Inevitably a display about historical automatons may work well for seventy-year-old retirees but could work less well for the four- to eight-year-old. Understanding the visitor groups to be targeted, their 
culture and interests, their information needs, and their attention spans, helps develop material that is relevant and engaging to target audiences.

\subsection{The space of the museum, the power of the object}

Above I discussed the challenge of creating digital-based interpretation at a museum level in a time overwhelmed with digital content. However, what needs to be recognized is the unique environment that the museum represents with its combination of object, story, and space. Most digital interaction is now via mobile devices and so untethered from specific location, spatially indeterminate, and geographically agnostic. The museum's fixed location and physical environments displaying collection objects interpreted with bespoke digital content provide potent opportunities for creating a unique experience.

The spatial arrangements allow exhibits to work as an installation. This follows from the concept of installation art in which a custom arrangement of objects and media organized together, frequently within a site-specific location, seeks to create a singular experience. Early precedents for this type of environment include the collaboration in 1958 between the architects Le Corbusier and Iannis Xenakis, and composer Edgard Varèse, in creating the Philips Pavilion for the 1958 World Expo in Brussels (Le Corbusier 1958). A purpose-designed pavilion hosted the multimedia production of Le Poème électronique using architectural-scale projection, spatialized sound, color, and film to create an 8-minute spectacle that was seen by 1.5 million visitors. In 1964 Charles and Ray Eames developed Think for IBM for their pavilion at the New York World Fair (Eames and Eames 1964). For this the audience were raised into a bespoke auditorium for a presentation playing across twenty-two screens synchronized together and introduced by a master of ceremonies dressed in a white dinner suit.

These two examples exist outside the museum context (from both a stylistic and a budget perspective) and do not directly feature objects, but they do illustrate the potential for creating one of Klein's scripted spaces dedicated to engaging spectacle, a theme that has been progressively adopted by museums and exhibitions ever since.

Within an environmental installation the collection objects play a role, becoming actors in the presentation of the story. Media and lighting can be used to highlight or amplify objects, bringing a theatricality or spectacle to the display. Using projection, sound, space, and media allows us to play with the natural scale of objects, elevating the presence they can bring to a gallery and manipulating our sense of them as objects.

\subsection{Case study: Watt's workshop, Science Museum, London}

Watt's workshop (Figures 1 and 2) is a long-standing exhibit at the Science Museum. It 


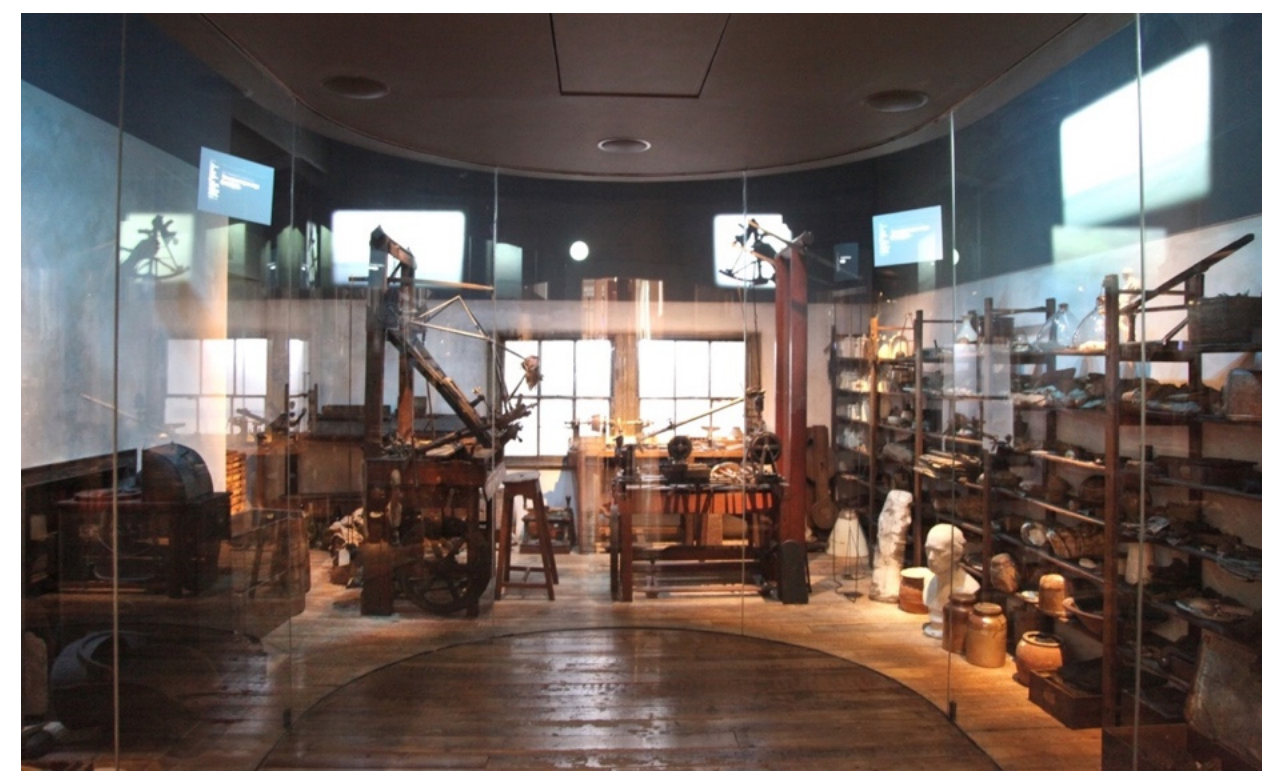

Figure 1: Watt's workshop in the Science Museum, London. Courtesy of Harmonic Kinetic.

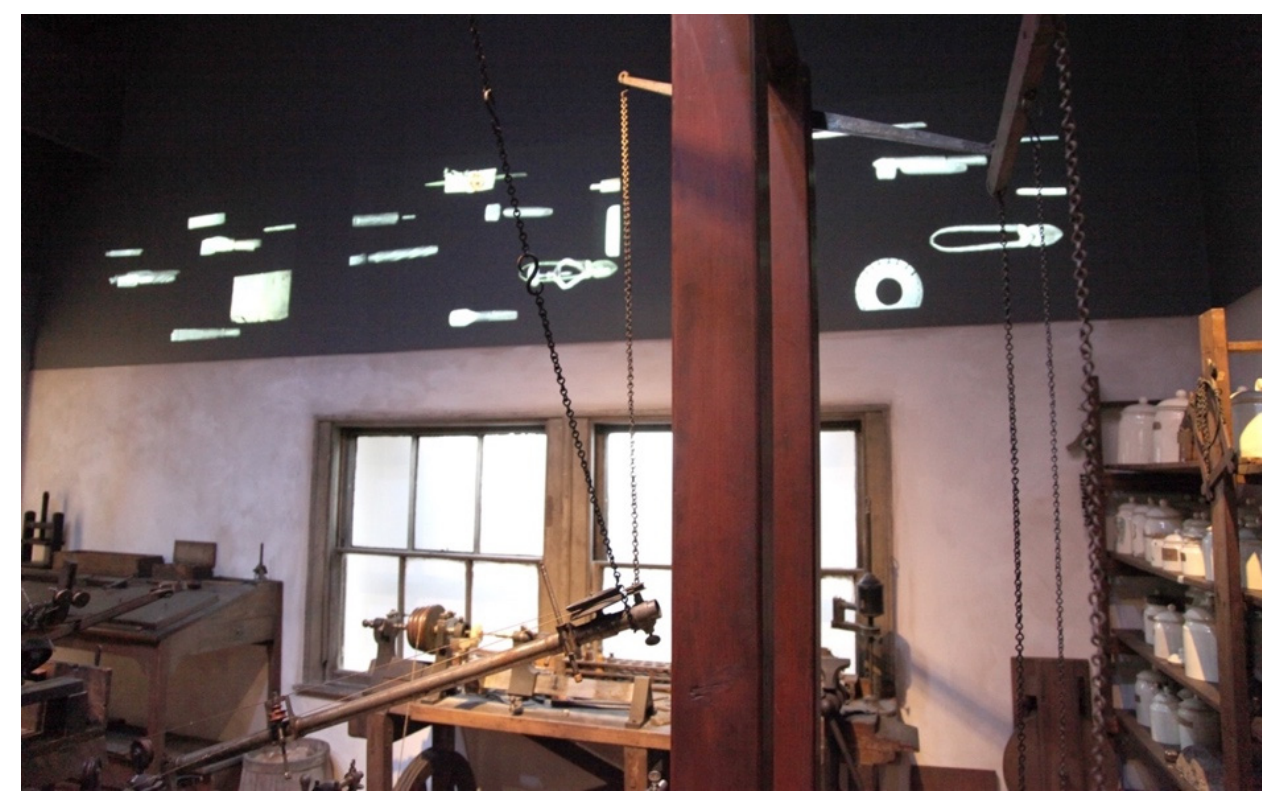

Figure 2: Watt's workshop machine and projections. Courtesy of Harmonic Kinetic.

is a re-creation of the workshop of the Scottish inventor James Watt, whose improvements to the steam engine were fundamental to the Industrial Revolution in the late eighteenth century. The museum space is filled with the inventions, tools, prototypes, and furniture taken from the original workshop at Watt's home in 
Staffordshire, which he used until his death in 1819. Watt was a serial entrepreneur and inventor creating everything from devices to count the revolutions of steam engines to machines that can mechanically copy sculptures.

The museum sought to create a media intervention to bring interpretation to the space. The creative brief was to bring the workshop to life, to give a sense of James Watt in his studio and in his life, obsessively pursuing and perfecting his ideas.

The final concept was to work with the list of 8400 objects from the workshop and use them to pick out key moments in Watt's life. In developing the installation, filmmaker Gilly Booth worked in residence with the collection, filming machines working and tools being used, and capturing the objects from different perspectives. This material was then edited together along with illustrations, animations, and soundscapes to create a multiple-projection installation around the workshop. The moving image material gives a fresh perspective on the workshop and Watt's life. Without being overly didactic, the environment and media illustrate his life through a selection of objects. They cover the death of his son Gregory from tuberculosis as well as his huge range of interests-not just innovations with steam technology but chemistry experiments with pottery glazes and building letter copying machines.

\subsection{Animation}

One immediate aim of interpretation exhibits is to bring an object to life in the eyes of the visitor, drawing their attention and building a curiosity that drives a stronger engagement with the collection. Hence, one of the primary tactics for activating and bringing a presence to an object is to literally bring animation or life to a display. This can be as simple as movement delivered through moving image, but it can be achieved through a range of different media: using physical movement to create kinetic displays that echo or illustrate the mechanical behaviors of the clock, using sound to re-create the workings of the clock, evoking the background sounds of the period, or using lighting to pick out details from the object.

As discussed above, an obvious starting point for animation with historical clocks and automatons is to present material that shows the clock working, using the full possibilities of filming and editing to give the visitor special views onto the object, zooming in to details to enhance the sense of the craftsmanship, and creating a sense of the clock as a world of micro-engineered mechanisms in perpetual motion. In this way it delivers key "missing" information to bring out an important visual comprehension of the object.

\subsection{Case study: standard time, Information Age, Science Museum, London}

An interpretive exhibit on standard time (Figures 3 and 4) is part of the Information 


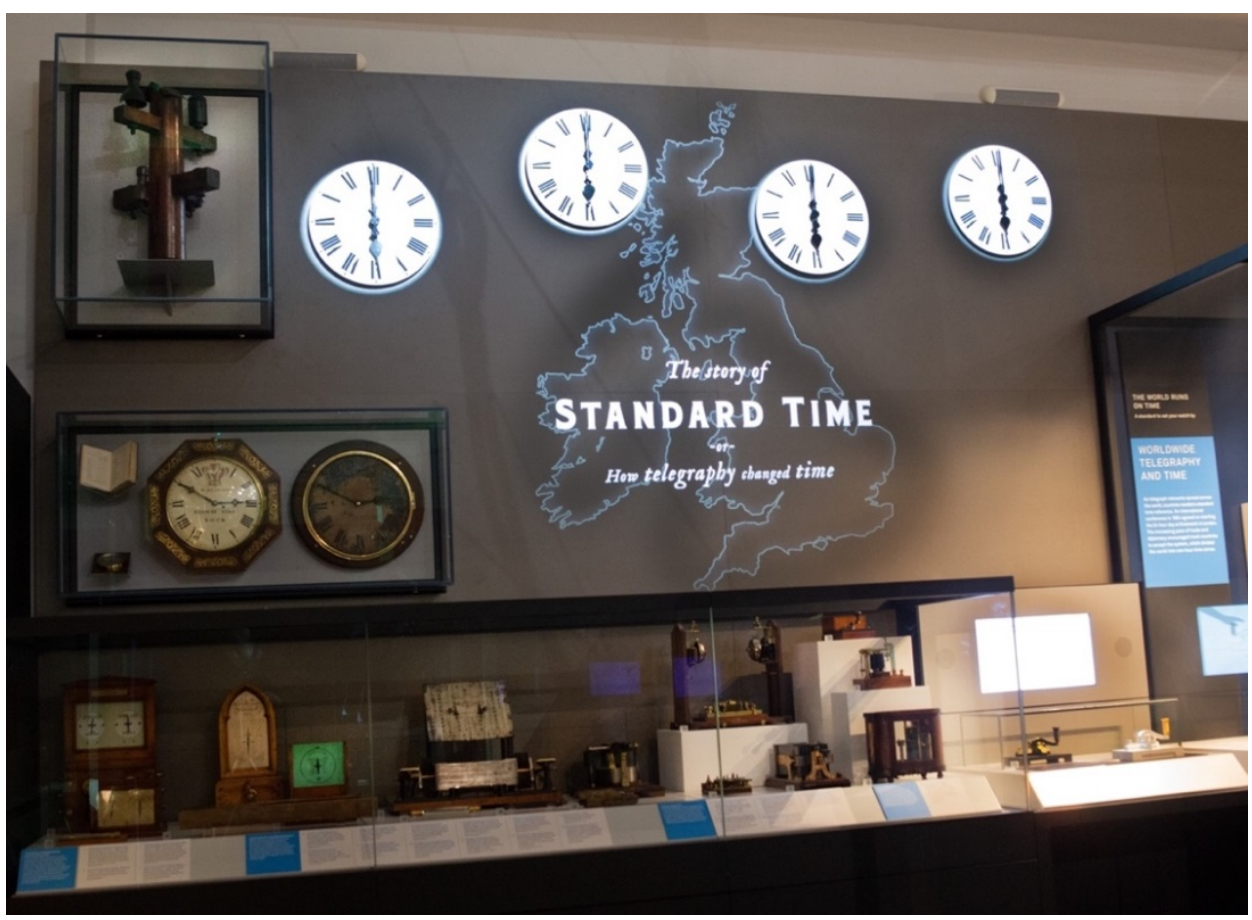

Figure 3: Telling the story of standard time. Courtesy of Harmonic Kinetic.

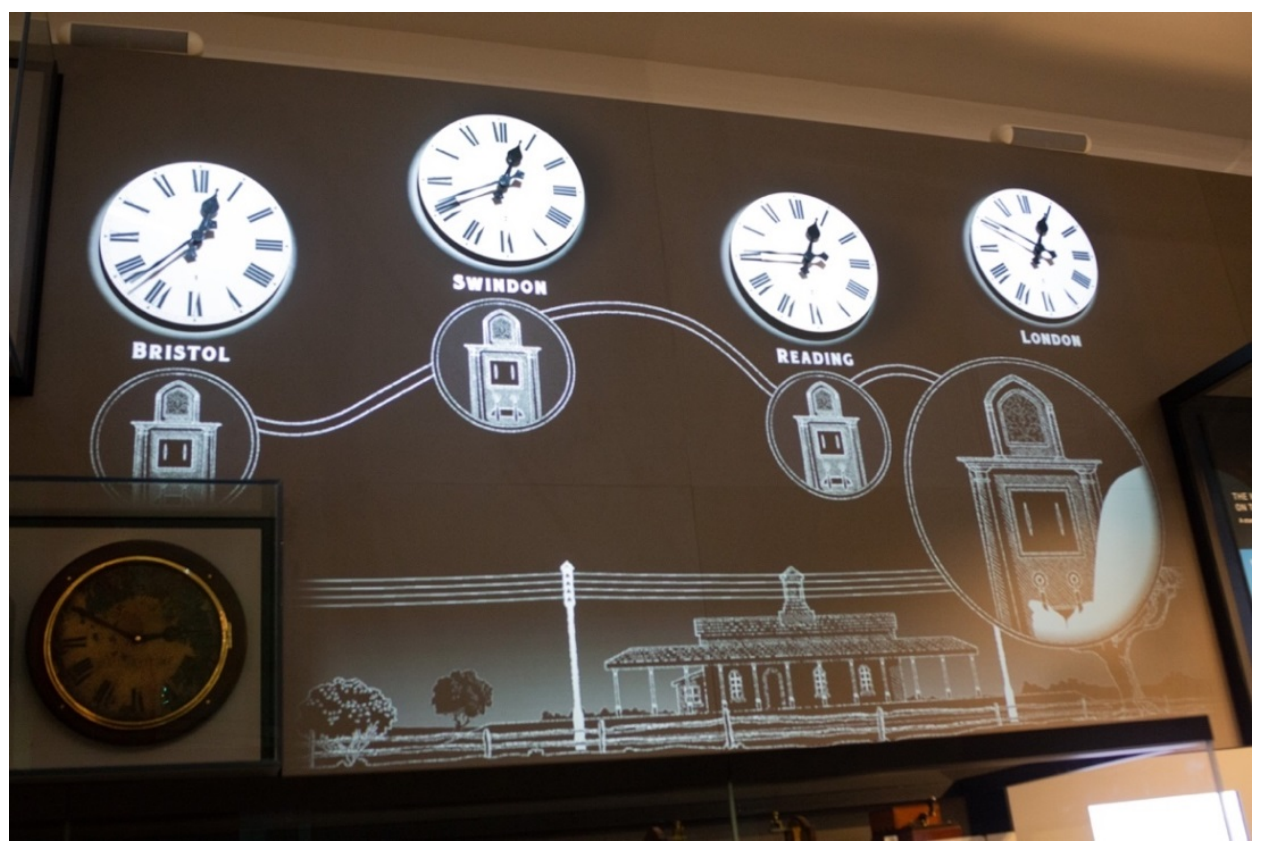

Figure 4: Detail of clock times before standard time. Courtesy of Harmonic Kinetic. 
Age gallery in the Science Museum. It is designed to work alongside a display of clocks and objects telling the story of the standardizing of time in Great Britain in the nineteenth century. Traditionally, clocks were set locally according to times of sunrise and sunset. However, the expansion of the railway and telegraph made these time differences increasingly complicated, and in 1840 railway companies started to adopt London time in stations across the country. By 1855 most public clocks in Britain were set to Greenwich Mean Time.

The exhibit uses three kinetic clocks (non-collection objects) as props across a projected map of Britain synchronized to the animated projection to reveal the time difference before time was unified. The projection recounts the story through an illustrated animation of the development of the railway and telegraph communications that led to the adoption of standard time.

In this instance the movement of the prop clocks is not describing the mechanics of the original objects but instead mirrors the form of the collection clocks to perform the part played by clocks in the story. As the designer, I wanted to give a strong graphic sense of the big cultural change when standard time was adopted nationally. The size of the projection brings a shift of scale to the story, allowing it to occupy a large wall space and bringing an animated presence to its corner of the gallery. The projection is set within a display of objects central to the story, including clocks and telegraphic communication devices.

\subsection{Visitor interaction}

Developing an exhibit that calls for hands-on interaction from the visitor can significantly enhance engagement with exhibition content. Interactive media can come in many different forms, from analogue physical interaction with mechanical interfaces, to digital formats such as video games. In essence, interaction offers the visitor an elevated agency, allowing them to navigate through information, comprehension, experimentation, and gameplay around an object. It shifts the experience from a passive mode of consumption to a participative one in which the visitor needs to make decisions, and demands an active level of cognitive reflection.

There are lots of different kinds of interactive content mechanisms and any kind of activity can potentially form the basis of an interactive exhibit. Collecting, building, quizzing, looking, guessing, drawing, and pointing all offer potential starting points. The design of the interaction alongside the choice of the interface becomes really important. Generally, a more tactile interface will lead to a more satisfying interaction.

\subsection{Case study: interactive models, Information Age, Science Museum, London}

Created by the London-based design studio Clay Interactive, the interactive models in 
the Information Age gallery (Figures 5 and 6) are a series of physical facsimiles of historical devices key to the development of modern communications, including a Morse key and a crystal radio set. The models themselves are interactive, with moving elements that work alongside a simple on-screen graphic animation, allowing the visitor to very clearly interact and explore how the devices worked.

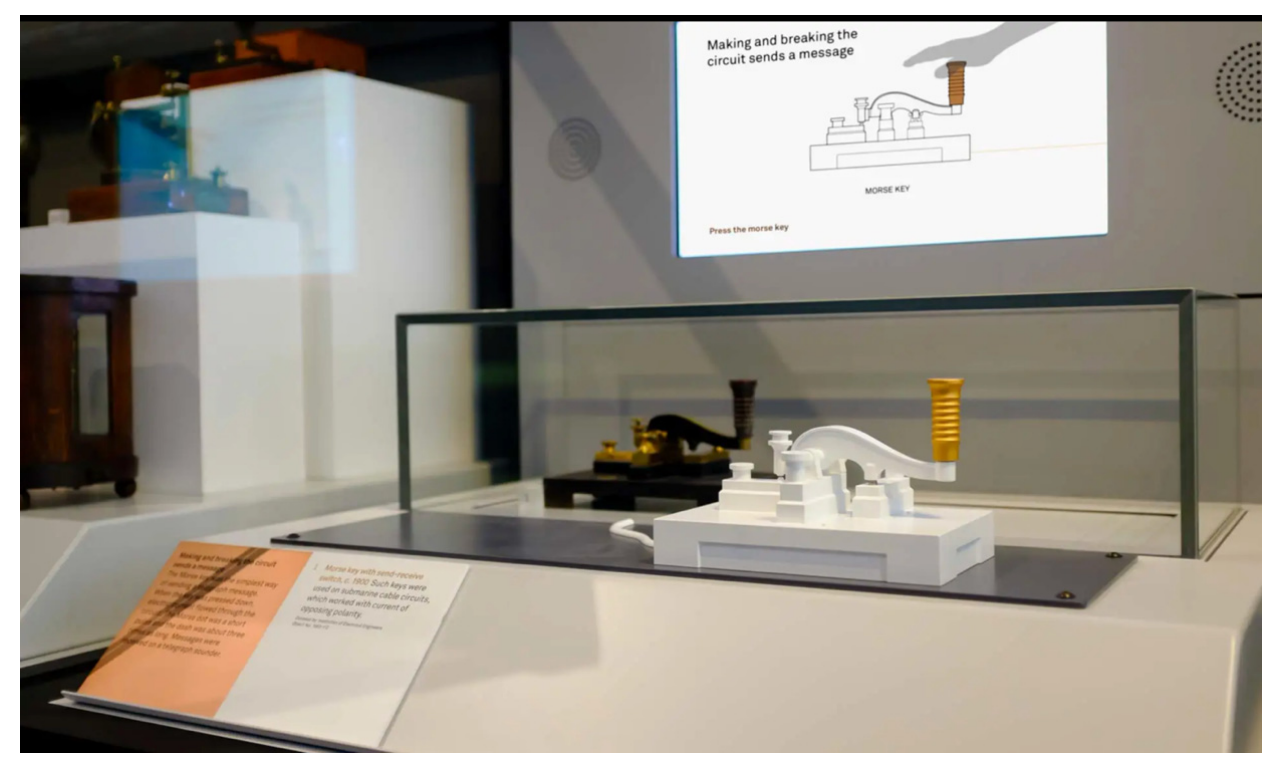

Figure 5: The Morse key interactive model alongside the original historical object. Courtesy of Clay Interactive.

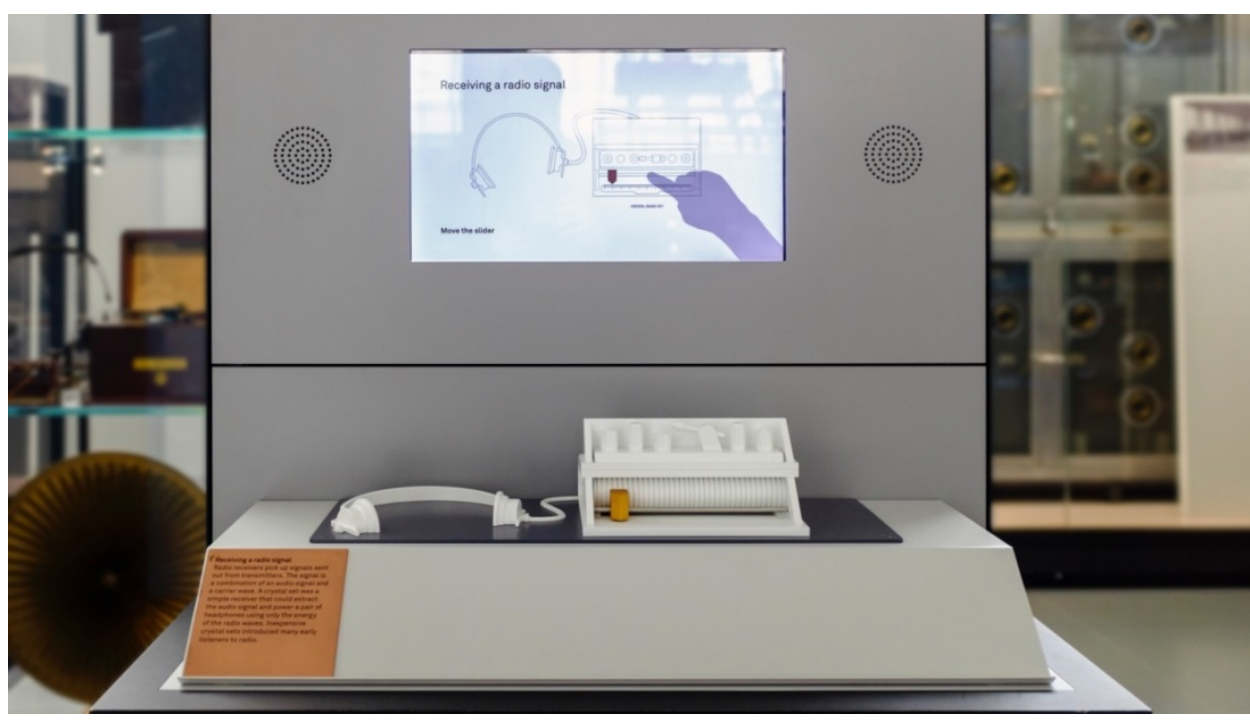

Figure 6: The crystal radio set interactive model. Courtesy of Clay Interactive. 
These designs very successfully communicate a complicated set of ideas and technical functionality for these devices. The physical models are unique and offer a tactile interactivity that is immediately engaging, giving the visitor real agency in exploring how they work. The interactive element for each model is carefully coded through the use of different colors. The design very carefully minimizes detail in the physical interfaces and the on-screen animations to prioritize the communication of how the devices work.

\subsection{Spectacle, wonder, surprise, humor, recognition}

An important aspiration in developing interpretation content for museums and exhibitions is to seek to build an emotional response or connection with visitors that works beyond information and facts. This should not be completely surprising, as this is what media creators are trying to achieve most of the time as film-makers, authors, actors, musicians, and so on. But perhaps it is less obvious when applied to interpreting immobile historical objects trapped in display cases.

Creating spectacle to evoke an emotional response has been consistently important to exhibition development. The spectacle of both artifacts and the audiences themselves was central to the attraction of the Great Exhibition of 1851, the precursor to the national museums of South Kensington in London (Bennett 2013). In contemporary times, the museum continues to offer an ideal venue for creating expanded and alternative models of spectatorship, competing with the traditions of cinema and television to create new and immersive ways to see the world (Griffiths 2008).

Many collection objects are naturally charged with spectacle-being big and old like a steam locomotive or a dinosaur skeleton helps. Historical clocks are more likely to achieve their spectacle through their age and beauty as crafted objects. Ultimately for the Palace Museum clocks it is the unique and extraordinary automated scenes revealed by the moving automatons that provide the real charm and wonder.

But wonder is only one kind of emotional response. Both surprise and humor in interpretation can positively challenge the formality of museum environments and allow us to look at objects in new ways. Unusual stories around objects can illuminate very different perspectives. The story of the Italian missionary first causing a stir in the Chinese imperial court by presenting two chiming clocks brings a human angle and a possibility for humor to a potentially distant historical narrative.

\section{Time, Culture and Identity: A Digital Exchange}

Time, Culture and Identity: A Digital Exchange (Figures 7 and 8) was a workshop held in Beijing that sought to share practice and explore a range of methodologies used by designers and developers in creating object interpretation exhibits. The two-day event brought together thirty experts, digital designers, and developers alongside curators, 


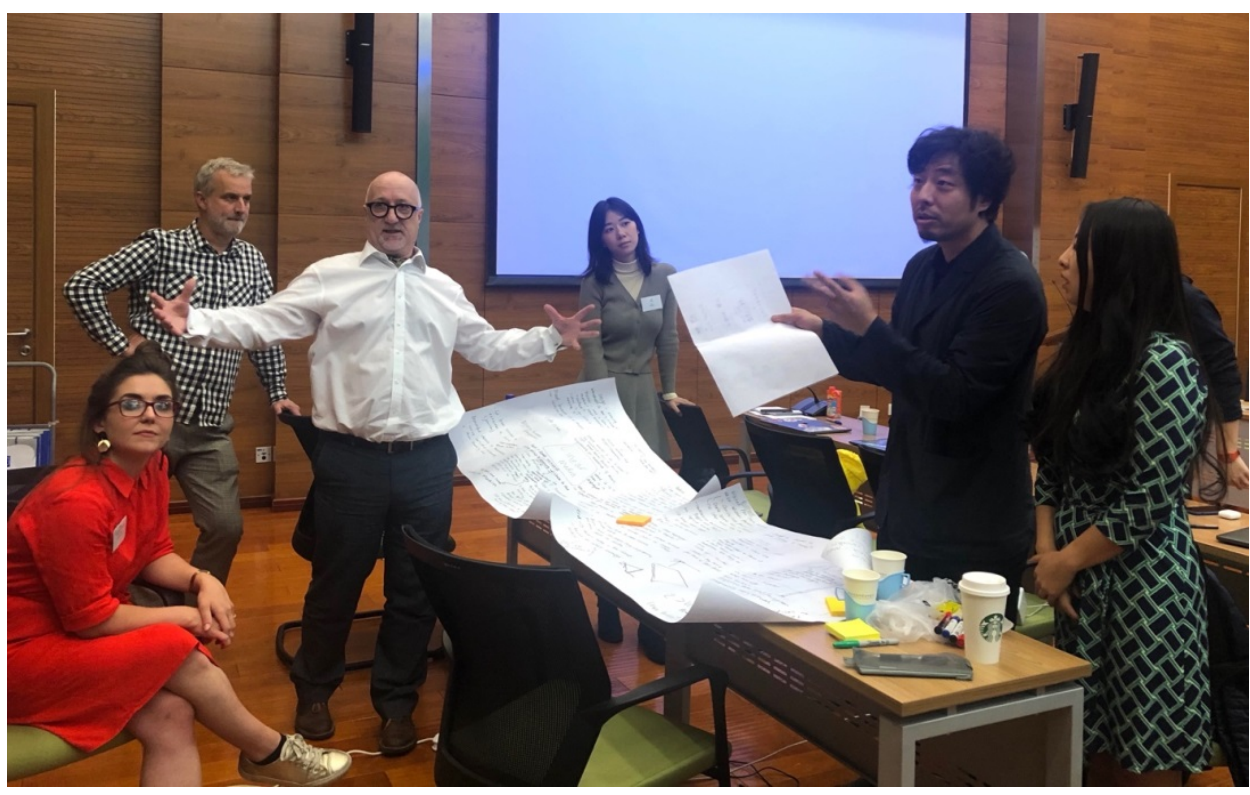

Figure 7: Small group discussion at the Time, Culture and Identity digital workshop.

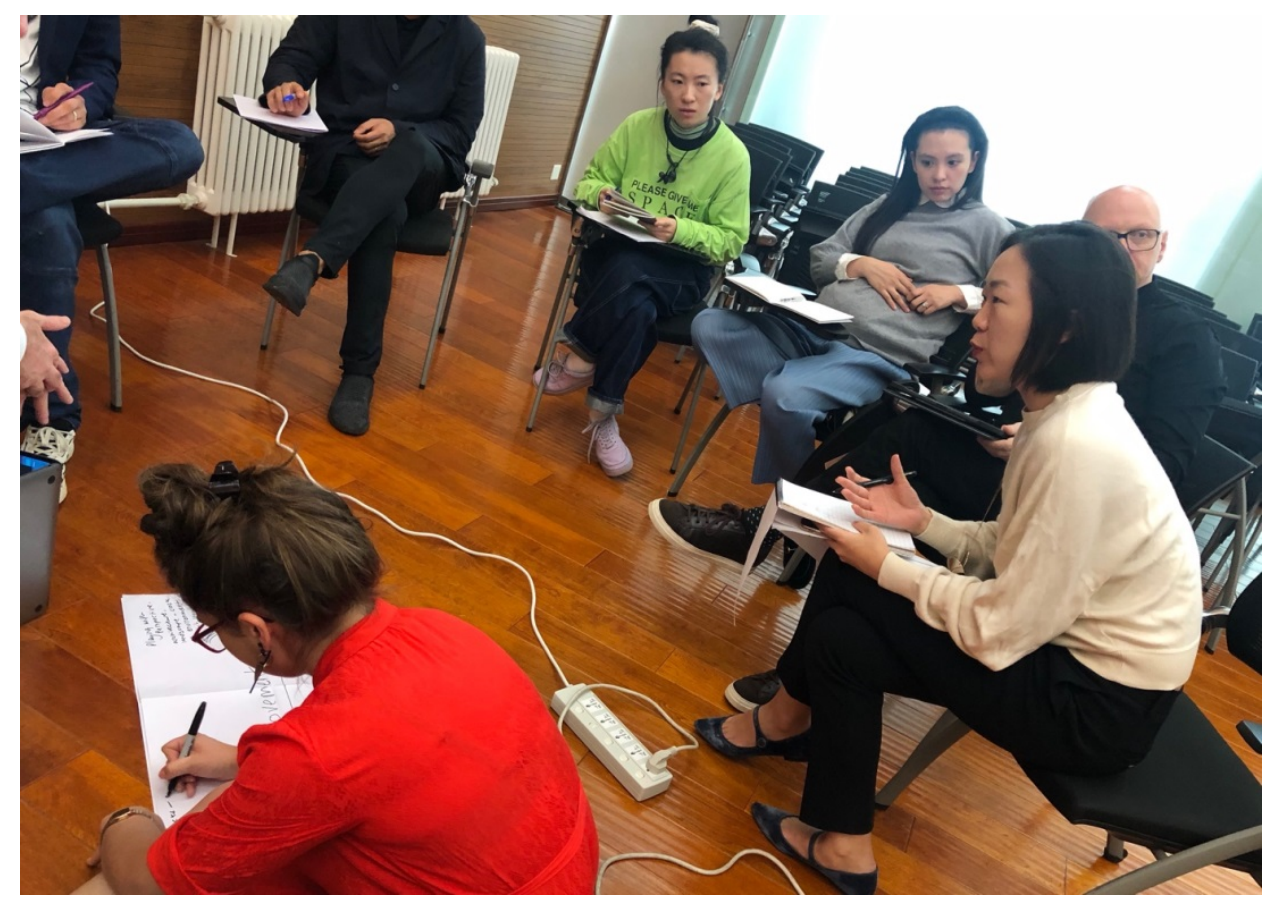

Figure 8: Small group discussion at the Time, Culture and Identity digital workshop. 
academics, and technologists. Creative practitioners gave short presentations showing recent projects alongside presentations on audience research in China and the UK and the mechanisms of the automatons in the imperial collection.

An important focus of the workshop was to explore an exhibit for a future temporary exhibition, Zimingzhong 自鸣钟: Clockwork Treasures from China's Forbidden City, to be held at the Science Museum in London.

\section{Creative sessions}

Two creative sessions were run in the digital workshop to consider different approaches to creating digital interpretation for the Country Scene clock from the Palace Museum collection. These sessions drew on codesign practices taken from an interaction design context and translated to work with a broader constituency of interdisciplinary participants.

Various methods were employed using the timeframe of a short active session for idea generation. This involved serial processes of opening up a field and closing it down, creating a workshop dynamic to draw out a wide range of ideas whilst enabling a number of these ideas to be taken forwards for further development.

The first session considered how broad themes and interest areas could identify concepts to be developed into an interpretive exhibit. The second creative session took these simple ideas from the first session and spent some time imagining how they could turn into a firmer range of exhibit proposals.

\subsection{First session}

The first creative session started with three short audience insights drawn from a comprehensive set of research carried out as part of the Time, Culture and Identity project, looking at audience engagement with the imperial clock collection of the Palace Museum (Chung 2020). These insights were purposefully oversimplified as a provocation for the exercise:

- Objects do not appeal to a general audience.

- Realization that the objects move significantly raises the audience's interest level.

- Universal interest is in the movement of the clocks and the fascination that this brings.

Alongside this was the clear constraint that the physical movement of the Country Scene clock would only be able to be demonstrated at the opening and closing of the exhibition, and so would not be part of the general exhibition.

Finally we reiterated a series of simple rules widely used for idea generation in workshops: 
1. One voice at a time.

2. Every idea is a good idea - wild and crazy ideas are very good.

3. No judging!

4. Quantity over quality.

5. Be playful.

6. Capture everything-Post-it notes (Figure 9), drawings, and so on.

These rules are intended to open up the scope of contributions, allowing participants to bring big ideas without needing to offer precise detail. Strong starting points can emerge when participants can relax everyday critical boundaries to play with an idea. The essential aspect for these sessions is to harness the dynamics of the group, applying its collective intelligence, imagination, and diverse perspectives to generate as broad a range of ideas as possible.

To bring some constraint, ideas were to be collected under four themes or story headings:

- The movement of the automatons (external)

- Design and making

- Trade

- Mechanics (internal)

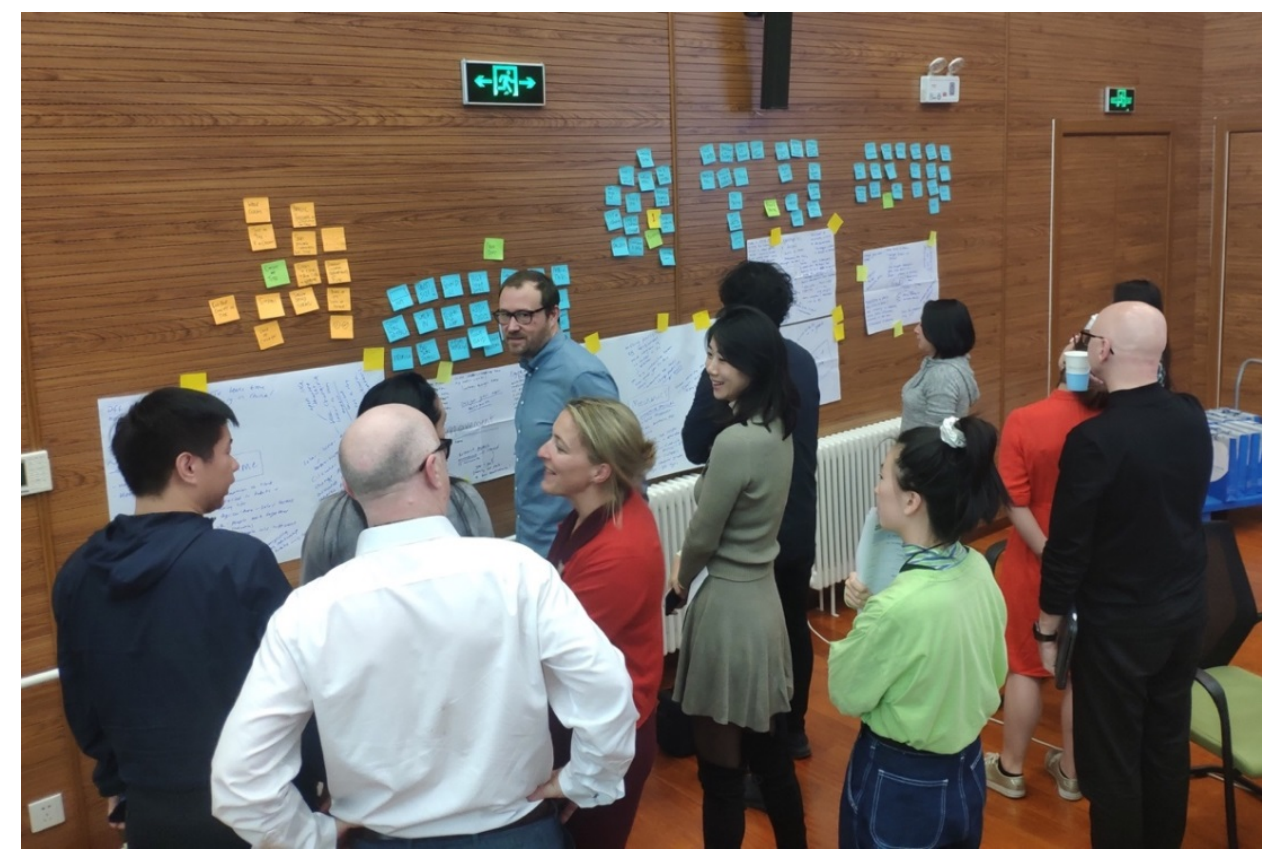

Figure 9: Reviewing a sea of Post-it notes in the digital workshop.

In smaller groups, ideas for these themes were discussed and recorded with text descriptions. At the end of this session the range of sketch ideas were distilled into a series 
of notes using simpler word descriptions to represent and classify the different concepts (Figure 10). Each of the groups then presented their ideas to the rest of the workshop.

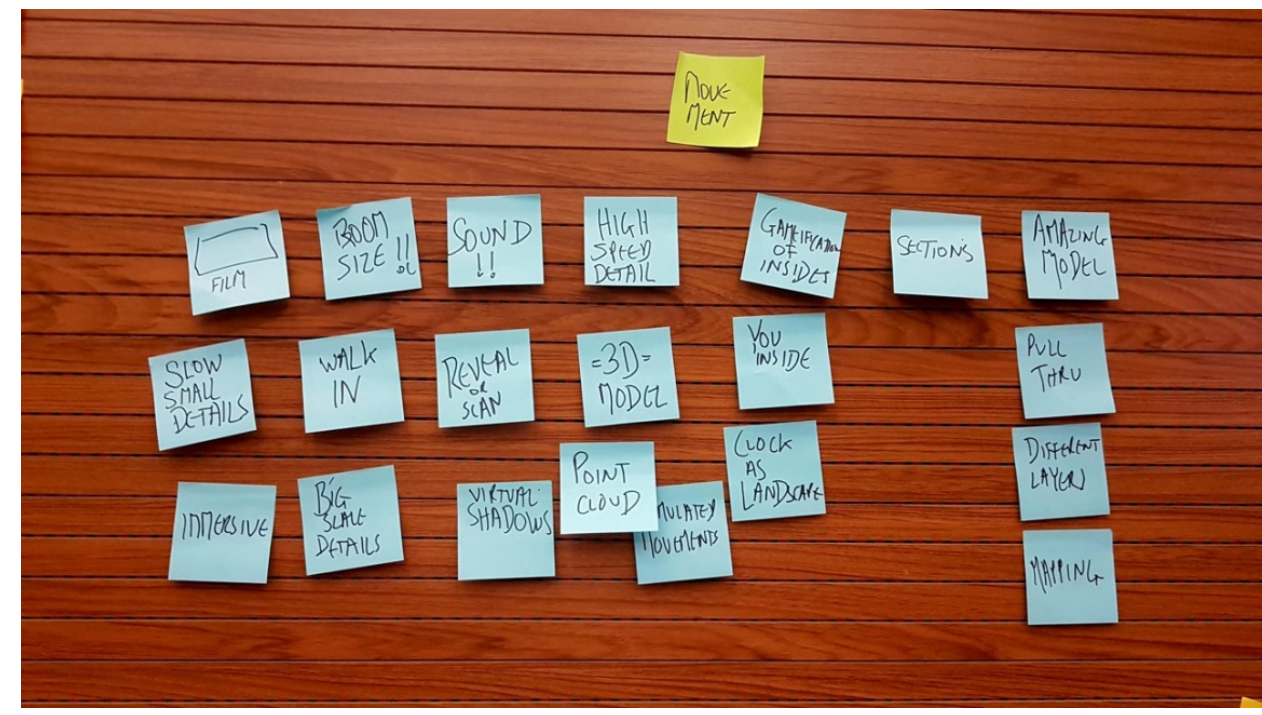

Figure 10: Simple text notes to represent each of the initial ideas focusing on the movement of the automatons.

\subsection{Second session}

The ideas from the first session were then developed through further discussion to work towards concepts for exhibits that can be visualized and illustrated. At this point the groups could start to filter, selecting and concentrating on the stronger and more interesting possibilities to develop. Visualization through sketching (Figure 11) is a very important stage of the idea development process, as it allows participants to consider and discuss the real material of the exhibit and start to bring some detail to its form, interaction, media, and so on.

A series of ideas emerged from these sessions that with development could become firmer proposals for an exhibit:

- Using a physical interface to let visitors build or assemble parts of the clock; using a digital medium to guide or reward the visitor.

- Bringing the automaton animals featured in the Country Scene clock to life, to relate the history and stories around the clock, for example, bringing out the "voice of the chicken."

- A play of scale in which visitors are shrunk to clock dimensions and can explore the workings at a room scale.

- Using a slow-reveal physical interface in which visitors can only see a part of the hidden clock interior at any point, perhaps like an X-ray scan, encouraging them to 
interactively explore the internal workings.

- Clock interiors projected within model spaces to explain the inner mechanical workings.

- Ask the watchmaker-using an AI and historical expertise to answer questions about the clocks and their history.

- An interactive sound environment to explore the clock workings and the sounds of different clocks.

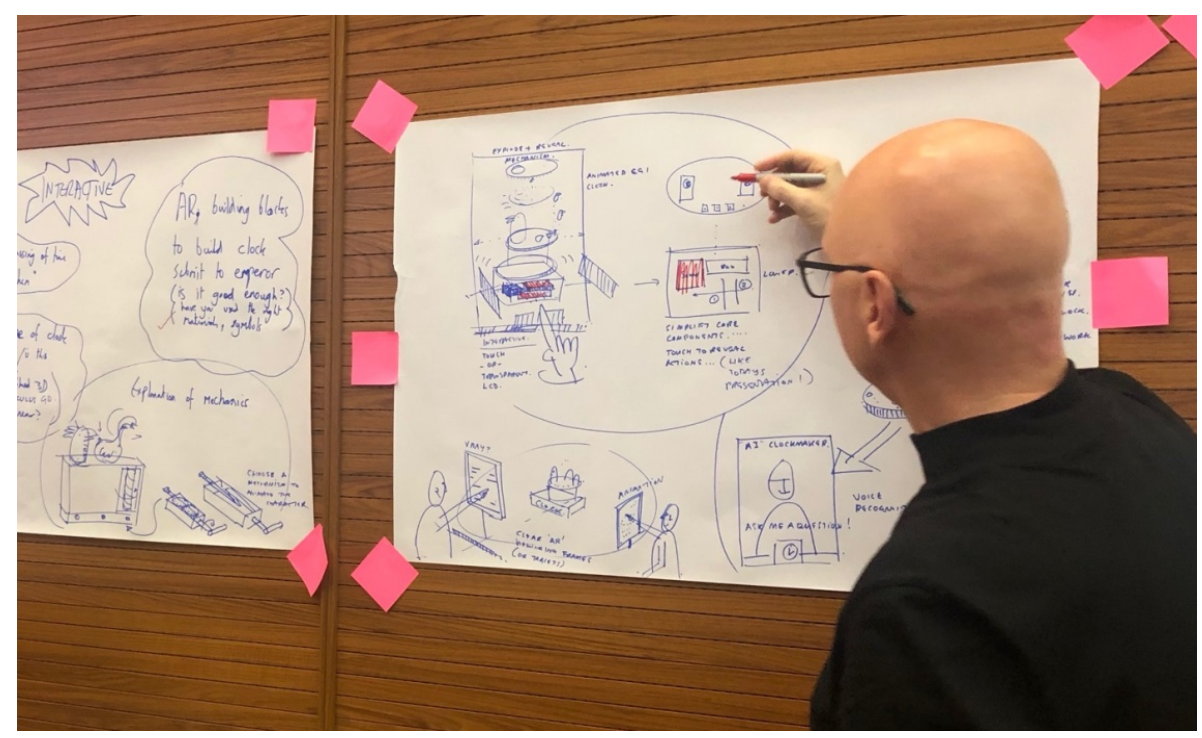

Figure 11: Sketching of exhibit proposals.

\section{Reflections from the digital exchange workshop}

The digital exchange workshop soundly demonstrated the potential for building a strong interdisciplinary platform and enabling diverse practitioners to work together. It proved that with a good format ideas can be cocreated and developed by small groups quickly.

This offers new methods for wider participation and codesign in exhibition interpretation content, a part of the exhibition process that has been traditionally siloed, with curators and developers working separately and at different stages.

Several factors worked well together to build this platform:

- Providing an opportunity for sharing important contextual information in presentations and discussions. Participants are given the opportunity to present their perspective and identify their priorities for any development.

- Defining the "content" to bring a clear focus for generating content ideas, in this case the single collection object the Country Scene automaton.

- Open creative sessions that encourage equal participation regardless of background or discipline. 
There were further important reflections from the workshop on the process of commissioning interpretive exhibits (Science Museum 2020). The Chinese creative practitioners who participated came from digital agencies in which their work is seen as new media art and is less prevalent in museum spaces. Their approach to digital experiences can bring a wide and open range of ideas and influences. By comparison the UK creatives were more likely to come from agencies working substantially in the museum and exhibition sector. In a bid to win a competitive pitch for a project, their responses to a creative brief are much more likely to be well designed and carefully "tailored" to visitor needs as defined by the museum. The suggestion is that while the current system for commissioning museum interpretational exhibits that prevails in the UK works and is well established, it could constrain the range of creative responses generated for new digital exhibits. New methods and commissioning approaches could allow a wider range of creative practitioners to be involved in the development of content, with benefits for greater diversity and more innovative outcomes.

The digital workshop in its prototype form readily suggests an alternative process for commissioning and developing new digital interpretational content, one that builds an enhanced level of dialogue and participation between all those involved.

\section{Conclusion}

Digital technology presents exciting potential for transforming the museum's job of bringing collection objects and their stories to life and widening their appeal for visitor groups. Emerging digital technologies offer us new forms of media, and these all come with strengths and weaknesses. A good understanding of the different types of media and what they can deliver empowers exhibition developers to make informed choices. Ultimately the most important stage in the process is to carefully consider the content that the interpretation reveals, what are the most engaging stories to be told, what does the visitor want to see but cannot, and what is missing in the static object. At this point the designer can consider the approach that fully exploits the unique setting of the museum environment and its available resources to create an exhibit that evokes a response and builds a connection with the audience. With the clocks of the imperial collection there is a clear need to show the automatons working, allowing visitors to see them in their full animated glory, the charm and variation of the movement, and the choreography of the clockwork. But for objects of time with such rich history, interpretation should go beyond and reveal more of the secrets of their life, the human (or animal) stories. If objects could speak, then the chicken that has been pecking away faithfully for nearly two hundred years in the "Gilt bronze clock with country scene and water automaton" from the imperial collection surely has some stories to tell.

Engagement and spectacle in its various forms has always been key to the museum 
experience. Working with new media in this context requires a level of constant innovation and experimentation with the opportunities to create these experiences. The digital exchange workshop suggested new methods for museums to work with creative practitioners to achieve this. Enhancing the participation in the process and building towards a better conversation between curators and designer-developers brings new possibilities for developing content collaboratively. It makes it easier to bring a wider range of creative practitioners into the development process, particularly those working outside the sector, to innovate and present an exciting new era for museum interpretation.

\section{Acknowledgment}

Thanks to the participants in Time, Culture and Identity: A Digital Exchange for their permission to use workshop images.

\section{References}

Bennett, T. 2013. The Birth of the Museum. Abingdon: Routledge.

Blyth, T. 2015. "Information Age? The Challenges of Displaying Information and Communication Technologies." Science Museum Group Journal (3). Last modified March 23, 2015. Accessed November 4, 2020. http://dx.doi.org/10.15180/150303.

Blyth, T., and A. Prugnon. 2015. "Storytelling the Information Age." In EVA London 2015: Electronic Visualisation and the Arts, 7-9 July 2015, London, UK, edited by K. Ng, J. P. Bowen, and N. Lambert. BCS, The Chartered Institute for IT.

Chung, C. 2020. "Time, Culture and Identity: Exploring Horological Collections with UK-China Museum Audiences." Chinese Annals of History of Science and Technology 4 (s): 82-102.

Eames, C., and R. Eames, dir. 1964. Think. New York: Film for IBM at the New York World Fair.

Griffiths, A. 2008. Shivers Down Your Spine. New York: Columbia University Press.

Klein, N. 2004. The Vatican to Vegas: A History of Special Effects. New York: New Press.

Le Corbusier. 1958. Le Poème électronique. Paris: Editions de Minuit.

Newzoo. 2019. “Newzoo Global Mobile Market Report 2019." Accessed September 4, 2020. https://newzoo.com/insights/trend-reports/newzoo-global-mobile-market-report-2019-lightversion.

Rounds, J. 2004. "Strategies for the Curiosity-Driven Museum Visitor." Curator: The Museum Journal 47 (4): 389-412.

Science Museum. 2020. "UK-China Collaboration in the Creative Industries: Reflections and Lessons Learned." Accessed September 4, 2020. https://www.sciencemuseumgroup.org.uk/ wp-content/uploads/2020/06/Time-Culture-Identity-Collaboration-guideline-A4-V6.pdf.

Wurmser, Y. 2019. “US Time Spent with Mobile 2019.” eMarketer. Accessed September 4, 2020. https://www.emarketer.com/content/us-time-spent-with-mobile-2019. 